\title{
TRANSPORTE DE ALTITUDE PARA O PICO DO CAMAPUÃ UTILIZANDO NIVELAMENTO TRIGONOMÉTRICO MÉTODO LEAP-FROG
}

Camapuã Hill altitude determination from leap-frog trigonometric leveling method

\author{
DANIEL PEROZZO DOS SANTOS ${ }^{1}$ \\ PEDRO LUIS FAGGION ${ }^{2}$ \\ LUIS AUGUSTO KOENIG VEIGA ${ }^{2}$ \\ ${ }^{1}$ Instituto Federal Catarinense - IFC - Campus Araquari \\ Rodovia BR 280 - km 27 - Cx. Postal 21 - CEP 89245-000 - Araquari - SC \\ ${ }^{2}$ Universidade Federal do Paraná -UFPR \\ Caixa Postal 19001 - CEP: 81531-990. Curitiba-PR \\ daniel.perozzo@ifc-araquari.edu.br; faggion@ufpr.br; kngveiga@ufpr.br
}

\begin{abstract}
RESUMO
O transporte de altitudes para pontos localizados em áreas de difícil acesso, como por exemplo, em regiões montanhosas, é um dos desafios da Geodésia, pois é necessária a transposição de grandes escarpas e vales, muitas vezes sem estradas de acesso, o que inviabiliza a utilização do nivelamento geométrico geodésico. Em função disso, técnicas modernas para a determinação de desníveis utilizando nivelamento trigonométrico vêm sendo estudadas, buscando-se precisão equivalente ao nivelamento geométrico, e com isso viabilizando o transporte de altitudes para estas regiões. Neste artigo realiza-se o transporte de altitude para o pico do Camapuã, na Serra do Mar paranaense, partindo-se de uma RN do Sistema Geodésico Brasileiro, localizada próxima à região. A metodologia utilizada é baseada no método Leap-Frog de nivelamento, com a realização de visadas de até aproximadamente $6000 \mathrm{~m}$, com desníveis de quase $1000 \mathrm{~m}$. São apresentados os resultados e uma avaliação do método.
\end{abstract}

Palavras-Chave: Geodésia; Altitudes; Nivelamento Trigonométrico.

\begin{abstract}
The orthometric height determination for points located in areas of difficult access, for example, in mountainous regions, is one of Geodesy challenges, because the transposition of great cliffs and valleys is necessary, often without access roads,
\end{abstract}


which makes unfeasible the use the geometric leveling. Modern height determination techniques from trigonometric leveling are being studied, seeking equivalent accuracy to geometric leveling and to make easy this task for these regions. In this paper is accomplished the height determination to the peak Camapuã, on Serra do Mar in the Paraná State. The methodology is based on the Leap-Frog trigonometric leveling, involving single sight lengths of about $6000 \mathrm{~m}$ with height differences of almost $1000 \mathrm{~m}$. The results and an evaluation of method are presented.

Keywords: Geodesy; Heights; Trigonometric leveling.

\section{INTRODUÇÃO}

Neste trabalho é realizado o transporte de altitude para o Pico do Camapuã, uma das maiores elevações do Estado do Paraná, localizado na Serra do Ibitiraquire, uma porção da Serra do Mar paranaense situada na divisa entre os municípios de Campina Grande do Sul e Antonina.

A altitude é transportada a partir de uma RN do Sistema Geodésico Brasileiro, localizada às margens da rodovia BR-116, nas proximidades da região de estudo. A metodologia utilizada é baseada no método de nivelamento Leap-Frog (KAHMEN e FAIG, 1988), que consiste na instalação da estação total num ponto qualquer de onde se tenha visada para os dois pontos entre os quais se queira determinar o desnível.

O método de nivelamento mais indicado para este trabalho seria o das visadas recíprocas e simultâneas, porém, devido à grande dificuldade de se transportar uma estação total e um tripé até o cume da montanha, optou-se pelo método Leap-Frog.

\subsection{Objetivos}

a) Avaliar o emprego do método Leap-Frog em condições extremas, com visadas de aproximadamente $6000 \mathrm{~m}$ e desníveis da ordem de $900 \mathrm{~m}$, situação em que a refração atmosférica tem um efeito significativo.

b) Determinar uma medida confiável de altitude para o Pico do Camapuã.

\subsection{Justificativa}

Técnicas de nivelamento trigonométrico, para determinação de desníveis de precisão, vêm sendo pesquisadas em regiões planas ou com pouca variação de altitude (GOMES, 2006; FAGGION, 2003; CEYLAN, 2005). Neste trabalho será avaliado o emprego do nivelamento trigonométrico, método leap-frog, envolvendo desníveis de quase 1000 metros. Neste caso, as variações das condições ambientais (pressão atmosférica, temperatura e umidade relativa do ar) são significativas ao longo da linha de visada, logo, devem ser consideradas.

Os valores de altitude até então conhecidos para as grandes elevações da Serra do Mar paranaense foram determinados, com raras exceções, utilizando barômetros, que, devido às condições encontradas nessas regiões, com variações significativas 
dos parâmetros ambientais ao longo do dia, proporcionam medidas altimétricas de baixa precisão. A conclusão deste trabalho proporcionará um valor confiável de altitude para o Pico do Camapuã.

\section{AMPARO CONCEITUAL DA METODOLOGIA UTILIZADA}

\subsection{Nivelamento Trigonométrico}

O nivelamento trigonométrico é um método indireto para a determinação de desníveis entre dois pontos. Um esquema básico pode ser observado na Figura 1.

Figura 1 - Nivelamento trigonométrico.

Adaptado de Kahmen e Faig (1988).

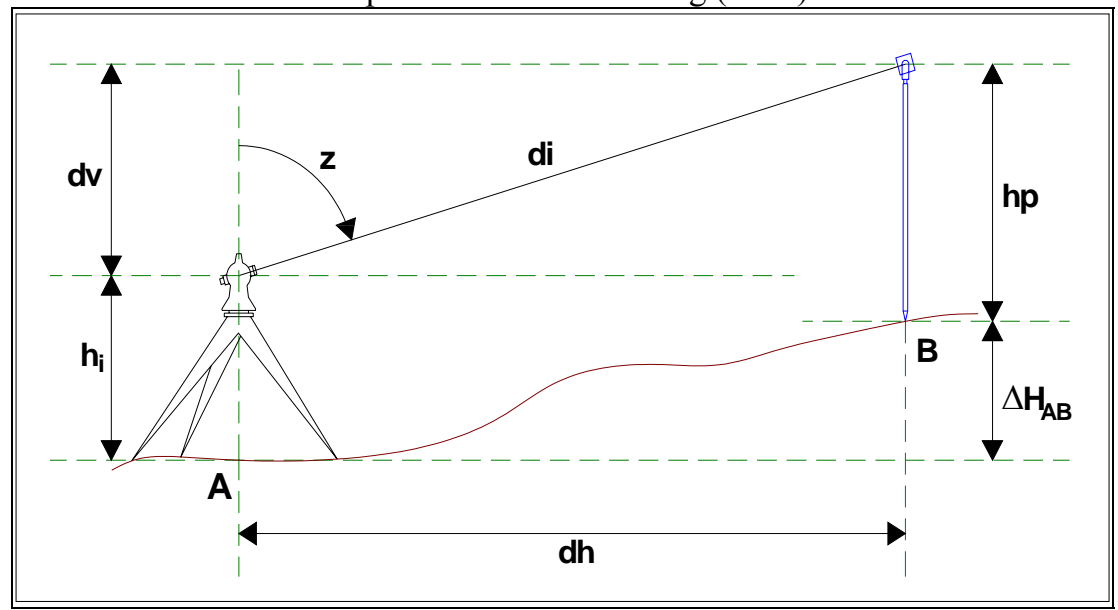

Onde:

di = distância inclinada;

dh $=$ distância horizontal;

$d v$ = distância vertical entre o centro ótico do equipamento e o centro do alvo;

$\mathrm{z}=$ distância zenital;

$\mathrm{hi}=$ altura do instrumento;

$\mathrm{hp}=$ altura do alvo ou prisma refletor;

$\triangle \mathrm{HAB}=$ desnível entre os pontos A e B.

Da figura tem-se a seguinte relação:

$$
d v+h i=h p+\Delta H_{A B}
$$

Isolando $\Delta H_{A B}$ obtém-se:

$$
\Delta H_{A B}=h i+d v-h p
$$

Bol. Ciênc. Geod., sec. Comunicações/Trab. Tecnicos Curitiba, v. 17, nº 2, p.295-316, abr-jun, 2011. 
Utilizando os conceitos da trigonometria para triângulo retângulo pode-se expressar $d v$ em função de $d i$ e $z$ que são grandezas medidas:

$$
d v=d i \cos (z)
$$

Substituindo (3) em (2) tem-se:

$$
\Delta H_{A B}=h i+d i \cos (z)-h p
$$

O maior inconveniente encontrado quando se pretende determinar desníveis de precisão utilizando nivelamento trigonométrico é a necessidade da medição da altura do instrumento e dos refletores (FAGGION et al, 2003). Essa medição é feita utilizando trenas e, mesmo considerando a hipótese de possuírem resolução milimétrica e serem calibradas, existem os erros que podem ser embutidos no processo de leitura, principalmente na medição da altura do instrumento, a qual não pode ser realizada na vertical.

Outros erros também estão embutidos no processo, como os erros instrumentais, curvatura terrestre e refração atmosférica. Em se tratando de grandes distâncias entre as RN's que se pretende determinar o desnível, que é a proposta deste trabalho, estes erros podem ter influência significativa.

A solução, portanto, é eliminar esses elementos do processo. Na literatura especializada existem metodologias desenvolvidas para minimizar estes erros, como, por exemplo, o método das visadas recíprocas e simultâneas (CEYLAN e BAYCAL, 2008; KAHMEN e FAIG, 1988) que minimiza os efeitos da curvatura terrestre e refração atmosférica, e o método Leap-Frog (KAHMEN e FAIG, 1988; CEYLAN et al, 2005), que elimina a necessidade da medição da altura do instrumento.

O método das visadas recíprocas e simultâneas é mais exato, porém está limitado a situações em que é possível instalar o instrumento sobre o ponto observado (MOREIRA, 2003). No presente trabalho, devido à dificuldade de se transportar uma estação total e um tripé até o pico da montanha, a utilização desse método foi descartada. Optou-se então pelo método Leap-Frog, que será descrito a seguir.

\subsubsection{Método Leap-Frog}

O princípio do método consiste em instalar a estação total entre os dois pontos dos quais se deseja obter a diferença de altitude, ao invés de colocá-la sobre um deles. Sobre esses pontos são instalados dois bastões com prismas refletores, que serão visados para obtenção das distâncias inclinadas e dos ângulos zenitais de ré e de vante (Figura 2). Esse procedimento faz com que a medida da altura do 
instrumento não seja necessária, pois é eliminada no processo de cálculos.

Cabe salientar que no método Leap-Frog, além da eliminação da medida da altura do instrumento, instalando-se a estação total na meia distância entre os pontos de ré e vante, os efeitos da curvatura terrestre e refração atmosférica serão minimizados. Porém, à medida que o tamanho da visada aumenta, mesmo instalando o equipamento no centro do lance, as diferenças nas condições atmosféricas entre os pontos de ré e de vante podem se tornar significativas. Kahmen e Faig (1988) recomenda um limite de $200 \mathrm{~m}$ para o comprimento das visadas.

Figura 2 - Nivelamento trigonométrico - método Leap-Frog. Adaptado de Moreira (2003).

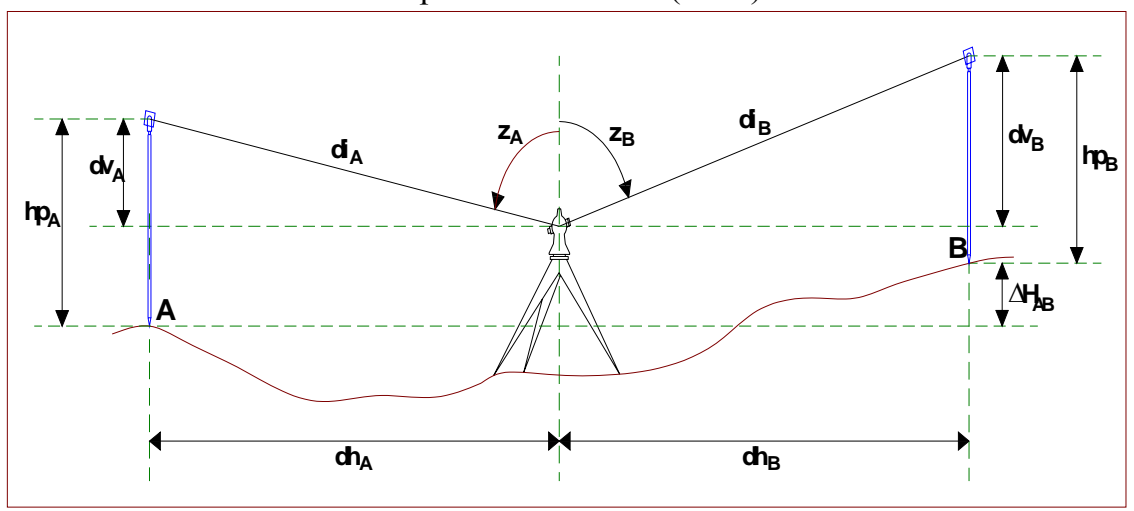

Onde:

$d v_{A}=$ Distância vertical em entre o centro ótico do equipamento e o centro do alvo em A;

$d v_{B}=$ Distância vertical em entre o centro ótico do equipamento e o centro do alvo em B;

$h p_{\mathrm{A}}=$ Altura do alvo em A;

$\mathrm{hp}_{\mathrm{B}}=$ Altura do alvo em B;

$\mathrm{di}_{\mathrm{A}}=$ Distância inclinada entre a estação e o alvo A;

$\mathrm{di}_{\mathrm{B}}=$ Distância inclinada entre a estação e o alvo B;

$\mathrm{dh}_{\mathrm{A}}=$ Distância horizontal entre a estação e o alvor A;

$\mathrm{dh}_{\mathrm{B}}=$ Distância horizontal entre a estação e o alvo B;

$\mathrm{z}_{\mathrm{A}}=$ Distância zenital A;

$\mathrm{Z}_{\mathrm{B}}=$ Distância zenital $\mathrm{B}$;

$\Delta H_{A B}=$ desnível entre os pontos A e $\mathrm{B}\left(\Delta H_{A B}=H_{B}-H_{A}\right)$.

Da Figura 2 tem-se:

Bol. Ciênc. Geod., sec. Comunicações/Trab. Tecnicos Curitiba, v. 17, nº 2, p.295-316, abr-jun, 2011. 


$$
\Delta H_{A B}=H_{B}-H_{A}=h p_{A}-d v_{A}+d v_{B}-h p_{B}
$$

Expressando $d v_{A}$ e $d v_{B}$ em função das grandezas medidas:

$$
\begin{aligned}
& d v_{A}=d i_{A} \cos \left(z_{A}\right) \\
& d v_{B}=d i_{B} \cos \left(z_{B}\right)
\end{aligned}
$$

Substituindo (6) e (7) em (5) tem-se:

$$
\Delta H_{A B}=h p_{A}-d i_{A} \cos \left(z_{A}\right)+d i_{B} \cos \left(z_{B}\right)-h p_{B}
$$

Uma simplificação encontrada na literatura (GOMES, 2006) consiste na utilização de bastões de igual altura para os pontos de ré e de vante. Deste modo, fazendo $h p_{A}=h p_{B}$, a Equação (5) é simplificada para:

$$
\Delta H_{A B}=d v_{B}-d v_{A}
$$

Expressando $d v_{A}$ e $d v_{B}$ em função das grandezas medidas, ou seja, substituindo as equações (6) e (7) em (9) obtém-se:

$$
\Delta H_{A B}=d i_{B} \cos \left(z_{B}\right)-d i_{A} \cos \left(z_{A}\right)
$$

Tratando-se de desníveis de $1^{\mathrm{a}}$ ordem utilizando apenas 1 lance, esta simplificação pode inserir um erro sistemático no processo, pois, mesmo utilizando bastões idênticos, do mesmo fabricante, podem existir pequenas diferenças em suas alturas, principalmente se forem bastões com algum tempo de uso, podendo ter ocorrido desgastes desiguais em suas ponteiras. Recomenda-se a medição acurada do comprimento dos bastões, por exemplo, utilizando um interferômetro LASER.

Realizando lances pares, assim como no nivelamento geométrico, inicia-se e termina-se o caminhamento com o mesmo bastão, eliminando a diferença entre as alturas dos bastões, como se fosse um erro de índice.

Trabalhando com distâncias acima de $200 \mathrm{~m}$ e com a estação total instalada fora do centro do lance, os efeitos da curvatura terrestre e refração atmosférica devem ser considerados.

\subsection{Curvatura Terrestre e Refração Atmosférica}

O caminho percorrido pelo sinal oriundo da estação total até o prisma refletor não é uma linha reta, e sim uma linha refratada, que sofre influência direta das variações nas condições atmosféricas entre o local onde está instalado o instrumento 
e o local onde está o prisma refletor.

Para uma atmosfera ideal, a densidade do ar diminui à medida que há um aumento na altitude, e consequentemente, o índice de refração do ar também diminui. Desta forma, segundo a Lei de Snell-Descartes, o caminho percorrido pela onda sofre um desvio, aproximando-se da normal na medida em que o índice de refração do meio aumenta, e vice versa.

O caminho percorrido pela onda sobre uma atmosfera ideal pode ser observado na Figura 3.

Figura 3 - Efeito da refração atmosférica sobre o sinal emitido.

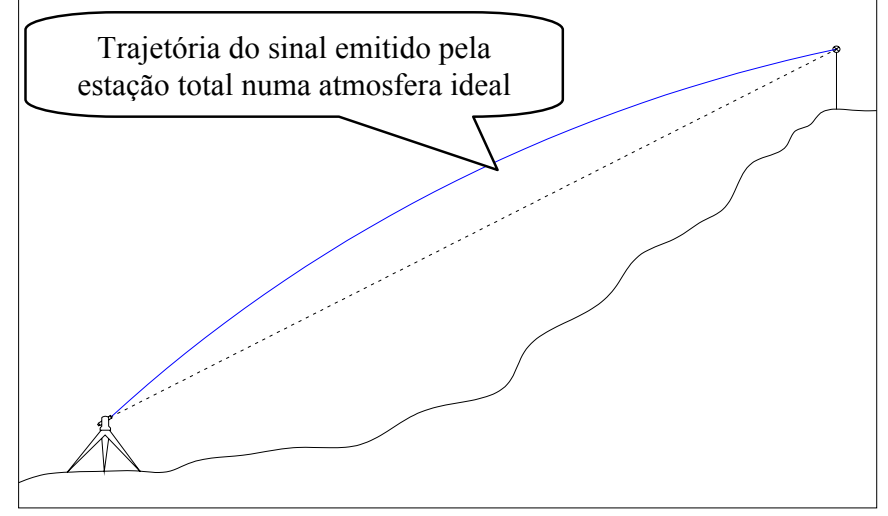

A curva gerada pelo efeito da refração pode ser considerada, numa primeira aproximação, como um arco de circunferência de raio r. Experiências tem comprovado que o valor deste raio é aproximadamente 8 vezes o raio da Terra, ou seja, $r \approx 8 \mathrm{R}$ (KAHMEN e FAIG, 1988), como mostrado na figura 4.

A relação entre o raio do sinal refratado $(\mathrm{r})$ e o raio terrestre $(\mathrm{R})$ é conhecida como coeficiente de refração da atmosfera terrestre, dado pela Equação (11) (KAHMEN e FAIG, 1988):

$$
k=\frac{1 / r}{1 / R}=\frac{R}{r}
$$

Onde:

$r=$ raio de curvatura do caminho percorrido pela onda;

$R=$ raio da Terra;

$k=$ coeficiente de refração.

Sendo $\mathrm{r} \approx 8 \mathrm{R}$, o valor de $k$ é aproximadamente 0,13 . Experiências mostram que, dependendo das condições ambientais, este valor varia entre $\pm 0,04$ ((KAHMEN e FAIG, 1988).

Bol. Ciênc. Geod., sec. Comunicações/Trab. Tecnicos Curitiba, v. 17, nº 2, p.295-316, abr-jun, 2011. 
Figura 4 - Correção da curvatura terrestre e refração atmosférica. Adaptado de Kahmen e Faig (1988).

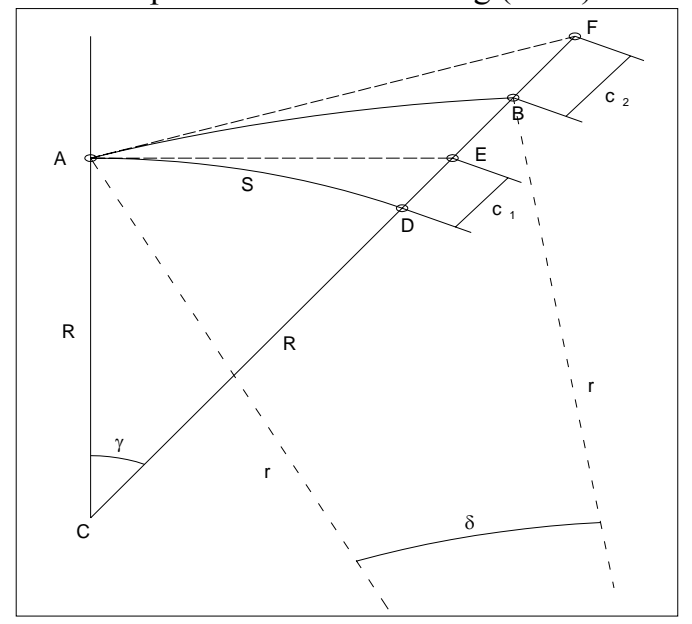

A Figura 4 exemplifica os efeitos da curvatura terrestre e refração atmosférica. Segundo Kahmen e Faig (1988), a correção da curvatura terrestre é dada pela Equação (12):

$$
c_{1}=\frac{S^{2}}{2 R}
$$

Onde:

$c_{1}=$ correção da curvatura terrestre;

$S=$ Distância horizontal entre os pontos;

$R=$ raio médio da Terra.

No quadro 1 pode-se observar os valores de correção da curvatura calculados para algumas distâncias definidas.

Quadro 1 - Correção da curvatura terrestre.

Fonte: Kahmen e Faig (1988).

\begin{tabular}{|c|c|c|c|c|c|c|}
\hline Distância & $100 \mathrm{~m}$ & $200 \mathrm{~m}$ & $500 \mathrm{~m}$ & $1000 \mathrm{~m}$ & $5 \mathrm{~km}$ & $10 \mathrm{~km}$ \\
\hline $\mathrm{c}_{1}$ & $0,8 \mathrm{~mm}$ & $3,2 \mathrm{~mm}$ & $2,0 \mathrm{~cm}$ & $7,9 \mathrm{~cm}$ & $1,96 \mathrm{~m}$ & $7,9 \mathrm{~m}$ \\
\hline
\end{tabular}

Segundo Kahmen e Faig (1988), a correção do efeito da refração atmosférica é dada pela Equação (13): 


$$
c_{2}=\frac{k S^{2}}{2 R}
$$

Analisando-se a Figura 4, pode-se observar que a correção da curvatura terrestre $\left(c_{1}\right)$ tem valor positivo e a correção da refração atmosférica $\left(c_{2}\right)$ tem valor negativo. Associando os dois efeitos, buscando uma única equação de correção, obtém-se:

$$
\begin{gathered}
c=c_{1}-c_{2}=\frac{S^{2}}{2 R}-\frac{K S^{2}}{2 R} \\
c=(1-k) \frac{S^{2}}{2 R}
\end{gathered}
$$

\subsection{Correção da influência dos efeitos ambientais sobre a distância medida}

Mudanças nas condições atmosféricas causam variações na velocidade de propagação das ondas eletromagnéticas, provocando, consequentemente, erros sistemáticos nas medidas de distância (FAGGION et al, 2003; RÜEGER, 1996). Para a correção do efeito da refração atmosférica sobre a distância medida normalmente utiliza-se a formulação que acompanha o manual do equipamento, onde é necessário o conhecimento da pressão atmosférica, temperatura e umidade relativa do ar.

\subsubsection{Cálculo da umidade relativa do ar}

Considerando a utilização de um psicrômetro analógico, é necessário efetuar o cálculo da umidade relativa do ar, que, segundo Rüeger (1996), pode ser realizado com as equações (16) à (18):

$$
e=E_{W}^{\prime}-0,000662 p\left(t-t^{\prime}\right)
$$

Onde:

$E_{W}^{\prime}=$ pressão de saturação do vapor d'água para a temperatura úmida (mbar);

$t=$ temperatura seca $\left({ }^{\circ} \mathrm{C}\right)$;

$t^{\prime}=$ temperatura úmida $\left({ }^{\circ} \mathrm{C}\right)$;

$p=$ pressão atmosférica (mbar);

$e=$ pressão parcial do vapor d'água (mbar).

$$
\begin{gathered}
E_{W}^{\prime}=10^{\left[\left(7,5 t^{\prime} /\left(273,3+t^{\prime}\right)\right)+0,7858\right]} \\
h=100 \frac{e}{E}
\end{gathered}
$$


Onde:

$e=$ pressão parcial do vapor d'água (mbar);

$E=$ pressão de saturação do vapor d'água para a temperatura seca (mbar);

$h=$ umidade relativa do ar $(\%)$;

O valor de $E$ pode ser calculado a partir da equação (17), apenas substituindo $E_{W}^{\prime}$ por $E$ e $t^{\prime}$ por $t$ (RÜEGER, 1996).

\subsection{Propagação de erros considerando a precisão nominal da estação total e estimativa do número de séries PD-PI}

Segundo Gomes (2006), para se determinar a precisão esperada para a distância vertical entre o centro óptico da estação total e o centro do alvo, é necessário aplicar a teoria da propagação de erros aleatórios.

Partindo da equação (3), $d v=d i \cos (z)$, e aplicando a teoria da propagação de erros, a precisão esperada para a distância vertical $d v$, considerando uma série de leituras PD-PI, é dada por (GOMES, 2006):

$$
\sigma_{d v}=\sqrt{\cos ^{2} z \sigma_{d i}^{2}+d i^{2} \operatorname{sen}^{2} z \sigma_{z}^{2}}
$$

Onde,

$z=$ ângulo zenital;

$d i=$ distância inclinada;

$\sigma_{d i}=$ precisão nominal linear da Estação Total;

$\sigma \mathrm{z}=$ precisão nominal angular da Estação Total;

Para determinar o número de séries PD-PI necessárias para se atingir uma precisão estimada na determinação do desnível $d v$, utiliza-se a equação (20), definido por Gomes (2006):

$$
N_{S}=\frac{\cos ^{2} z \sigma_{d i}^{2}+d i^{2} \operatorname{sen}^{2} z \sigma_{z}^{2}}{\sigma_{d v}^{2}}
$$

Onde:

$N_{S}=$ número de séries PD-PI.

\section{PREPARAÇÃO DOS INSTRUMENTOS}

\subsection{Verificação, retificação e calibração das Estações Totais}

Para se obter medidas de precisão é imprescindível trabalhar com equipamentos confiáveis, que sejam verificados periodicamente, e se necessário, retificados e calibrados. A Universidade Federal do Paraná, através do Curso de 
Pós-Graduação em Ciências Geodésicas, conta com uma estrutura completa para esta finalidade.

Buscando realizar a verificação da componente angular da estação total, utilizou-se um colimador de ajuste (Figura 5), seguindo a metodologia descrita por Faggion (2006).

Figura 5 - Colimador e Estação Total no processo de verificação em laboratório.
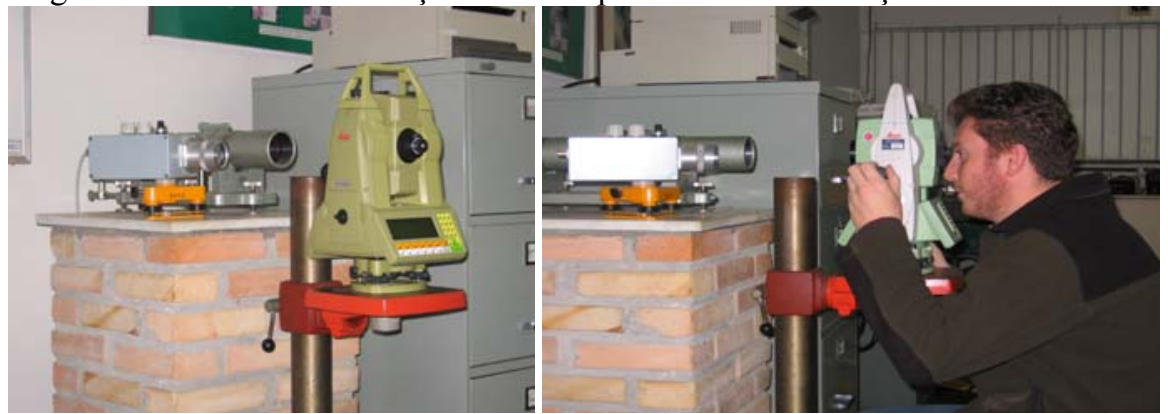

\subsection{Calibração dos bastões}

Os bastões utilizados nos levantamentos foram calibrados com o auxilio de um sistema de calibração instalado no LAIG (Laboratório de instrumentação Geodésica), que consiste num conjunto de trilhos nivelados e um interferômetro LASER. Este sistema foi implantado com a finalidade de calibrar miras verticais de ínvar, e tem a capacidade de determinar medidas de comprimento com precisão de $10^{-6} \mathrm{~m}$. Na Figura 6 pode-se observar os detalhes do sistema instalado no laboratório.

Figura 6 - Sistema Interferométrico utilizado na calibração.
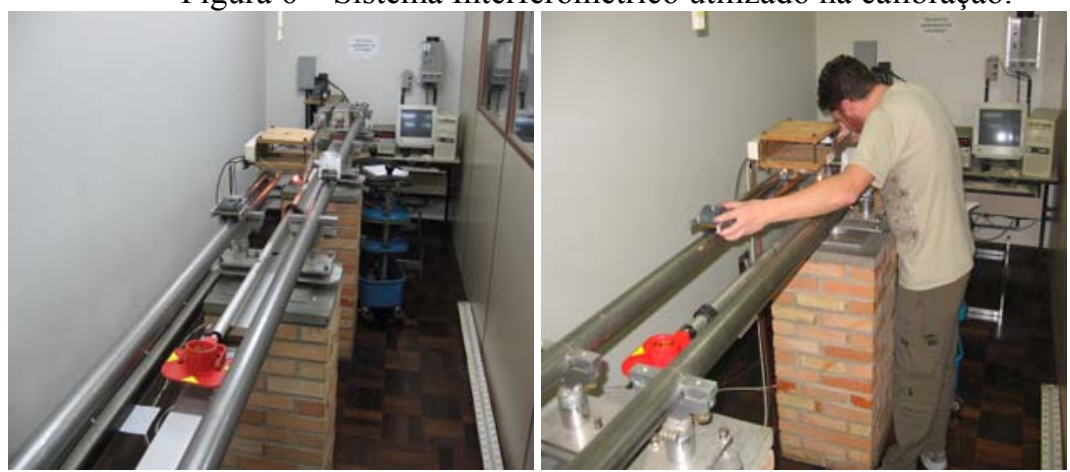

O sistema é dotado de um carrinho que se desloca sobre os trilhos, onde está instalado um microscópio utilizado para realizar as pontarias. Para garantir

Bol. Ciênc. Geod., sec. Comunicações/Trab. Tecnicos Curitiba, v. 17, nº 2, p.295-316, abr-jun, 2011. 
pontarias sempre verticais, o microscópio é dotado de um nível de bolha tubular altamente preciso. O procedimento adotado é a medição da ponta e o topo do bastão, e subtraindo uma leitura da outra se obtém seu comprimento. Recomenda-se a realização de várias leituras, obtendo-se uma média.

No nivelamento trigonométrico, utilizando estação total, existe a necessidade de instalação de um prisma refletor sobre o bastão. Em função disso, optou-se pela calibração do conjunto bastão+prisma, eliminando qualquer erro sistemático oriundo de diferenças entre a adaptação do bastão e o prisma. No processo de calibração, a pontaria é realizada no centro ótico do prisma e na ponta do bastão (Figuras 7 e 8), desta forma se obtendo diretamente a altura do prisma em relação à ponta do bastão.

Figura 7 - Medidas realizadas no centro óptico do prisma refletor.
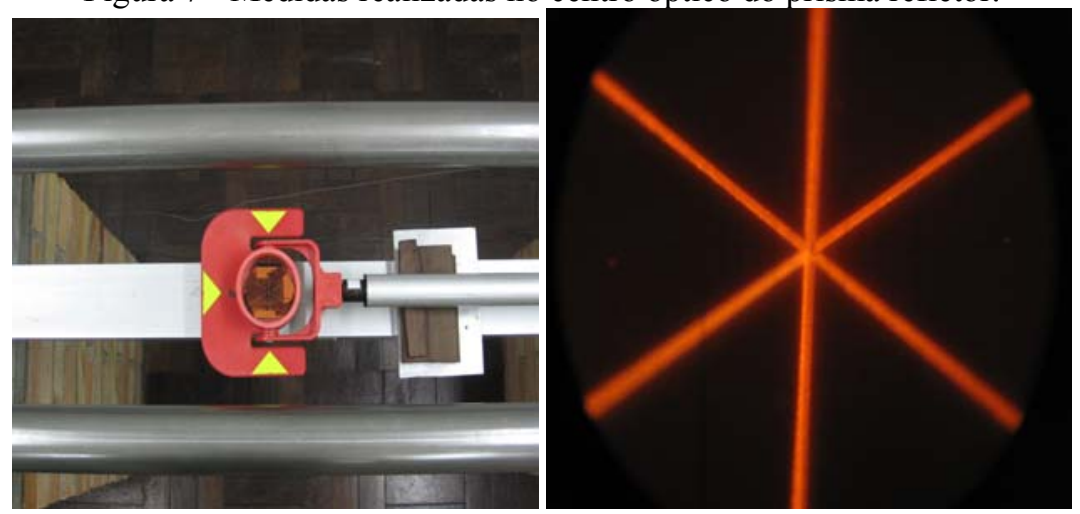

Figura 8 - Detalhe das pontarias na extremidade do bastão.
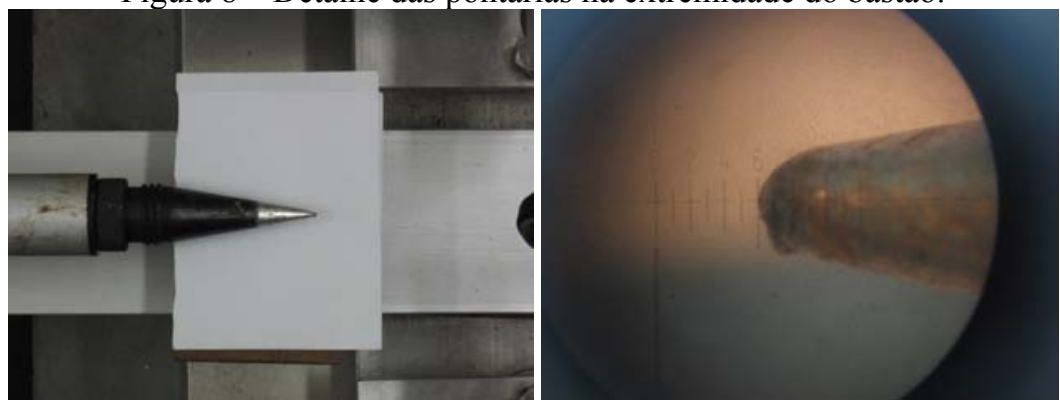

Bol. Ciênc. Geod., sec. Comunicações/Trab. Técnicos Curitiba, v. 17, no 2, p.295-316, abr-jun, 2011. 


\section{LEVANTAMENTO EXPERIMENTAL, CENÁRIO, EQUIPAMENTOS E PROCEDIMENTOS}

Para possibilitar o fechamento de um circuito de nivelamento, optou-se pela implantação de um ponto auxiliar que viabilizasse a realização de visadas tanto para o Pico do Camapuã, quanto para outros cumes localizados nas proximidades, com o intuito de dar continuidade em trabalhos futuros. Este novo marco também foi implantado às margens da BR116, a uma distância de aproximadamente $5,2 \mathrm{Km}$ ao norte da RN2045N. Devido à distância, foi necessária a implantação de um ponto intermediário, uma vez que não foi possível realizar o nivelamento no mesmo dia. Estes foram denominados de Aux01 e Aux02, como pode ser visto na figura 9.

Figura 9 - Área de estudos. FONTE: Google Earth.

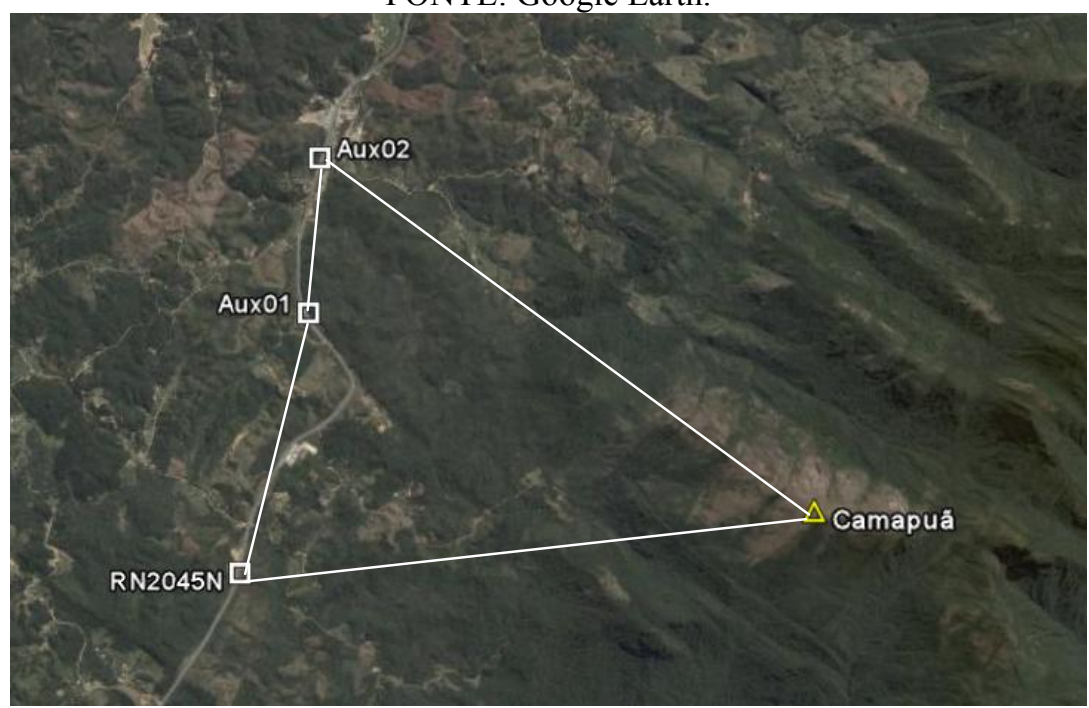

Realizou-se, ao longo da rodovia, nivelamento trigonométrico utilizando o método Leap-Frog, sendo duas seções de nivelamento e contranivelamento, uma entre a RN2045N e ponto Aux01, e outra entre os pontos Aux01 e Aux02 (Figura 9). Para ambas as seções foram necessários 4 lances de nivelamento.

Para este nivelamento utilizou-se a estação total Leica TC2003, com precisão linear de $1 \mathrm{~mm}+1 \mathrm{ppm}$ e angular de 0,5 '. Tomou-se o cuidado de realizar seções pares, terminando o caminhamento com o mesmo bastão que iniciou, eliminando a diferença de altura entre os bastões. Também foram coletados os parâmetros ambientais (pressão atmosférica, temperatura e umidade relativa do ar) para a correção dos efeitos das condições meteorológicas nas medidas de distância. Para calcular os valores de correção em ppm (partes por milhão) utilizou-se a formulação

Bol. Ciênc. Geod., sec. Comunicações/Trab. Tecnicos Curitiba, v. 17, nº 2, p.295-316, abr-jun, 2011. 
fornecida pelo fabricante, contida no manual do equipamento.

Para um maior rendimento no processo do nivelamento, utilizou-se visadas extremas, ou seja, a estação total não foi necessariamente instalada no centro de cada lance, com o comprimento das visadas variando entre 50 e $900 \mathrm{~m}$, extrapolando o limite recomendado de $200 \mathrm{~m}$. Neste caso, os efeitos da curvatura terrestre e refração atmosférica devem ser considerados.

Utilizando a equação (20), determinou-se o número necessário de séries de leituras PD-PI para se atingir alta precisão $(3 \mathrm{~mm} \sqrt{\mathrm{K}})$, considerando a precisão nominal do equipamento. Para uma distância inclinada de até $900 \mathrm{~m}$, utilizando a estação total Leica TC2003, é necessária apenas uma série PD-PI para atingir a precisão estimada de $3 \mathrm{~mm} \sqrt{\mathrm{K}}$, porém, realizaram-se entre 4 a 5 séries tendo em vista que as condições encontradas em campo não eram as ideais.

A partir da RN2045N e Aux02 realizou-se nivelamento trigonométrico até o pico Camapuã, também baseado no método Leap-Frog, mas agora em condições extremas, com a estação total instalada a menos de $100 \mathrm{~m}$ do ponto de ré, nas margens da rodovia, e o ponto de vante sendo o cume da montanha, com distâncias inclinadas de aproximadamente $6000 \mathrm{~m}$ (Figura 10). As condições desfavoráveis do terreno não possibilitaram a instalação da estação total no centro do lance.

Figura 10 - Realização de pontarias no o cume do Camapuã.

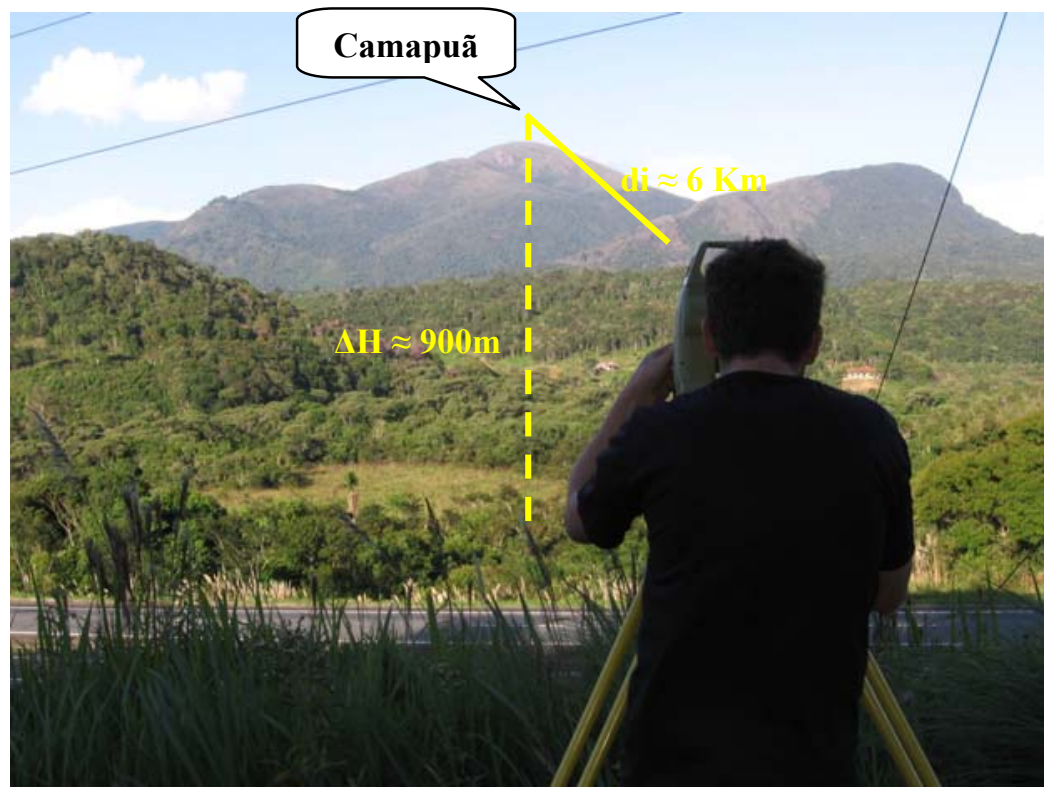

Para este tipo de nivelamento não foi possível utilizar a estação total Leica 
TC2003, que é a mais precisa, pois a mesma utiliza portadora LASER na faixa do infravermelho, e possui capacidade para medir distâncias com um único prisma de, no máximo, 3500m. Utilizaram-se as estações totais Leica TCRA1205, com precisão angular de 5" e linear de $2 \mathrm{~mm}+2 \mathrm{ppm}$, e Leica TCR407power, com precisão angular de 7" e linear de $2 \mathrm{~mm}+2 \mathrm{ppm}$. Ambas operam, além do infravermelho, com portadora $L A S E R$ na faixa do visível, e possuem capacidade de medir distâncias de até $10 \mathrm{~km}$ com apenas 1 prisma refletor.

Devido à distância entre a estação total e o prisma localizado no cume da montanha, de aproximadamente $6 \mathrm{~km}$, tem-se um valor de correção da distância para o local onde está a estação total e outro para o topo da montanha. A única solução encontrada foi calcular a média entre o valor da correção perto da estação total e o valor no cume da montanha. Em Rüeger (1996, pg 117), há um exemplo de correção de longa distância utilizando este mesmo artifício da média.

Pretendendo-se atingir fechamento de alta precisão $(3 \mathrm{~mm} \sqrt{\mathrm{K}})$, aplicou-se a equação (20) para calcular o número necessário de séries de leituras PD-PI, considerando a precisão nominal do equipamento e utilizando o comprimento aproximado das visadas a serem realizadas. Verificou-se que seria impraticável realizar esta quantidade de séries, então, calculou-se também o numero de séries necessárias para atingir as precisões esperadas de $6 \mathrm{~mm} \sqrt{\mathrm{K}}, 8 \mathrm{~mm} \sqrt{\mathrm{K}}$ e $12 \mathrm{~mm} \sqrt{\mathrm{K}}$, como pode ser observado no Quadro 2.

Quadro 2 - Número de séries PD-PI para se atingir a precisão esperada.

\begin{tabular}{|l|c|c|c|c|c|}
\hline \multicolumn{6}{|c|}{ Estação Total Leica TCRA 1205 } \\
\hline Seção & $\begin{array}{c}\text { Comprimento } \\
\text { da Visada }\end{array}$ & $3 \mathrm{~mm} \sqrt{\mathrm{K}}$ & $6 \mathrm{~mm} \sqrt{\mathrm{K}}$ & $8 \mathrm{~mm} \sqrt{\mathrm{K}}$ & $12 \mathrm{~mm} \sqrt{\mathrm{K}}$ \\
\hline $\begin{array}{c}\text { RN2045N - } \\
\text { Camapuã }\end{array}$ & $6000 \mathrm{~m}$ & 392 & 98 & 56 & 25 \\
\hline $\begin{array}{c}\text { RNAux02 - } \\
\text { Camapuã }\end{array}$ & $6300 \mathrm{~m}$ & 412 & 103 & 58 & 26 \\
\hline \multicolumn{6}{|c|}{ Estação Total Leica TCR 407 Power } \\
\hline Seção & $\begin{array}{c}\text { Comprimento } \\
\text { da Visada }\end{array}$ & $3 \mathrm{~mm} \sqrt{\mathrm{K}}$ & $6 \mathrm{~mm} \sqrt{\mathrm{K}}$ & $8 \mathrm{~mm} \sqrt{\mathrm{K}}$ & $12 \mathrm{~mm} \sqrt{\mathrm{K}}$ \\
\hline $\begin{array}{c}\text { RN2045N - } \\
\text { Camapuã }\end{array}$ & $6000 \mathrm{~m}$ & 768 & 192 & 108 & 48 \\
\hline $\begin{array}{c}\text { RNAux02 - } \\
\text { Camapuã }\end{array}$ & $6300 \mathrm{~m}$ & 807 & 202 & 114 & 51 \\
\hline
\end{tabular}

Realizou-se o maior número possível de séries, considerando as dificuldades encontradas em campo, como a longa caminhada até o cume, ventos, nebulosidade e precipitações. Também foram executadas seções de nivelamento e contranivelamento, possibilitando a verificação do erro de fechamento de seção. $\mathrm{O}$ 
Quadro 3 mostra o número de séries realizadas e o equipamento utilizado em cada seção de nivelamento.

Quadro 3 - Número de séries PD-PI realizadas.

\begin{tabular}{|c|c|c|c|}
\hline \multirow{2}{*}{ Seção } & & $\begin{array}{c}\text { Número de } \\
\text { Séries PD-PI }\end{array}$ & $\begin{array}{c}\text { Equipamento } \\
\text { utilizado }\end{array}$ \\
\hline RN2045N - \\
Camapuã & Nivelamento & 31 & TCR 407 Power \\
\cline { 2 - 4 } RNAux02 - & contra & 17 & TCRA 1205 \\
Camapuã & Nivelamento & 21 & TCRA 1205 \\
\cline { 2 - 4 } & contra & 21 & TCRA 1205 \\
\hline
\end{tabular}

\section{RESULTADOS}

Para as seções de nivelamento ao longo da rodovia, RN2045N-Aux01 e Aux01-Aux02, conseguiu-se fechamento melhor que $3 \mathrm{~mm} \sqrt{\mathrm{K}}$, ou seja, de alta precisão conforme a resolução $\mathrm{PR} \mathrm{n}^{\circ} 22$ (IBGE, 1983). Na primeira seção, entre a RN2045N e a RNAux01, o erro de fechamento foi de $1 \mathrm{~mm} \sqrt{K}$. Já na segunda seção, entre as RN's Aux01 e Aux02, o erro de fechamento foi de $3 \mathrm{~mm} \sqrt{\mathrm{K}}$.

Com relação ao nivelamento realizado entre as RN's localizadas às margens da rodovia e o cume do Camapuã, os resultados são apresentados na sequência de quadros a seguir. O Quadro 4 apresenta a média e desvio padrão das séries de observações PD-PI realizadas para cada seção de nivelamento. Já no Quadro 5 encontram-se os resultados da seção de nivelamento e contranivelamento executada entre a RN2045N e o Pico do Camapuã, e no Quadro 6, da seção entre a RN Aux02 e o Pico do Camapuã.

Quadro 4 - Média dos desníveis e desvio padrão.

\begin{tabular}{|c|c|c|c|c|}
\hline \multirow{2}{*}{ Seção } & & $\begin{array}{c}\text { Número de } \\
\text { Séries PD- } \\
\text { PI }\end{array}$ & $\begin{array}{c}\text { Desnível } \\
(\mathrm{m})\end{array}$ & $\begin{array}{c}\text { Desvio Padrão } \\
(\mathrm{m})\end{array}$ \\
\hline \multirow{2}{*}{$\begin{array}{c}\text { RN2045N } \\
\text { - Camapuã }\end{array}$} & Nivelamento & 31 & 895,58080 & 0,0573 \\
\cline { 2 - 5 } $\begin{array}{c}\text { RNAux02 } \\
\text { - Camapuã }\end{array}$ & Nivelamento & 17 & 895,59059 & 0,0880 \\
\cline { 2 - 5 } & contra & 21 & 910,63158 & 0,0718 \\
\hline
\end{tabular}


Quadro 5 - Resultados do nivelamento entre a RN 2045N e Camapuã.

\begin{tabular}{|c|c|c|c|c|}
\hline \multicolumn{2}{|c|}{$\begin{array}{l}\text { Seção: } \\
\text { RN2045N - Camapuã } \\
\end{array}$} & Sem correções & $\begin{array}{c}\text { Com Correção } \\
\text { da Curvatura }\end{array}$ & $\begin{array}{c}\text { Com correção da } \\
\text { Curv. e Refr. }(K=0,13)\end{array}$ \\
\hline \multirow{3}{*}{ Desnível (m) } & Nivelamento & 893,20683 & 895,93553 & 895,58080 \\
\hline & Contra & 893,21670 & 895,94531 & 895,59059 \\
\hline & Média & 893,21176 & 895,94042 & 895,58569 \\
\hline \multicolumn{2}{|c|}{ Erro cometido (mm) } & $-9,88$ & $-9,79$ & $-9,80$ \\
\hline \multicolumn{2}{|c|}{ Distância Nivelada (m) } & 5992,540 & & Tolerância de \\
\hline \multicolumn{2}{|c|}{ Distancia Contra-Nivelada (m) } & 6117.600 & & Erro permitido (mm) \\
\hline \multicolumn{2}{|c|}{ Média (m) } & 6055,070 & & 9,84 \\
\hline
\end{tabular}

Quadro 6 - Resultados do nivelamento entre a RN Aux02 e Camapuã.

\begin{tabular}{|c|c|c|c|c|}
\hline \multicolumn{2}{|c|}{$\begin{array}{l}\text { Seção: } \\
\text { Aux02 - Camapuã } \\
\end{array}$} & Sem correções & $\begin{array}{l}\text { Com Correção } \\
\text { da Curvatura } \\
\end{array}$ & $\begin{array}{c}\text { Com correção da } \\
\text { Curv. e Refr. }(\mathrm{K}=0,13)\end{array}$ \\
\hline \multirow{3}{*}{ Desnível (m) } & Nivelamento & 908,01510 & 911,02255 & 910,63158 \\
\hline & Contra & 907,99205 & 910,99344 & 910,60810 \\
\hline & Média & 908,00358 & 911,00799 & 910,61984 \\
\hline \multicolumn{2}{|c|}{ Erro cometido (mm) } & 23,05 & 29,11 & 23,48 \\
\hline \multicolumn{2}{|c|}{ Distância Nivelada (m) } & 6199,129 & & $\begin{array}{l}\text { Tolerância de } \\
12 \mathrm{~mm} \sqrt{K}\end{array}$ \\
\hline \multicolumn{2}{|c|}{ Distancia Contra-Nivelada (m) } & 6199,063 & & Erro permitido (mm) \\
\hline \multicolumn{2}{|c|}{ Média (m) } & 6199,096 & & 29,88 \\
\hline
\end{tabular}

Para a seção de nivelamento entre a RN2045N e o cume do Camapuã conseguiu-se um fechamento dentro da tolerância de $4 \mathrm{~mm} \sqrt{\mathrm{K}}$, o que pode ser classificado como desnível de precisão segundo a resolução PR n²22 (IBGE, 1983).

Bol. Ciênc. Geod., sec. Comunicações/Trab. Tecnicos Curitiba, v. 17, nº 2, p.295-316, abr-jun, 2011. 
Já para a seção entre a RNAux02 e o cume da montanha, o fechamento foi de $12 \mathrm{~mm} \sqrt{\mathrm{K}}$ (para fins topográficos segundo a PR n²2).

Foi possível fechar um circuito de nivelamento, como pode ser visto na Figura 9. No Quadro 7, encontram-se os erros de fechamento considerando três situações: nivelamento sem nenhuma correção, somente com a correção da curvatura, e com a correção da curvatura e refração.

Quadro 7 - Erro de fechamento de circuito.

\begin{tabular}{|c|c|c|c|}
\cline { 2 - 4 } \multicolumn{1}{c|}{} & Sem correções & $\begin{array}{c}\text { Com Correção } \\
\text { da Curvatura }\end{array}$ & $\begin{array}{c}\text { Com correção da } \\
\text { Curv. e Refr. (K=0,13) }\end{array}$ \\
\hline $\begin{array}{c}\text { Erro de } \\
\text { fechamento } \\
(\mathrm{mm})\end{array}$ & $-346,77$ & $-32,03$ & $-70,53$ \\
\hline
\end{tabular}

Teoricamente, o erro de fechamento considerando a combinação das correções da curvatura e da refração deveria ter o menor valor em módulo, mas na prática não foi o que ocorreu. Isso pode ser atribuído, principalmente, aos efeitos da refração atmosférica nas visadas, levando-se em consideração as condições extremas em que se realizou o nivelamento.

Buscando aplicar os conceitos de ajustamento de observações, tendo em vista que foi possível o fechamento de um circuito de nivelamento, realizou-se um ajustamento utilizando o método dos correlatos (GEMAEL, 1994).

Os desníveis utilizados no cálculo do ajustamento são os corrigidos da curvatura terrestre e refração atmosférica. Os valores dos desníveis sem correções e os somente corrigidos da curvatura terrestre são apresentados unicamente para comparação.

Como o fechamento das seções entre as RN's ao longo da rodovia foi significativamente melhor que o fechamento das seções até o cume do Camapuã, utilizar o inverso das distâncias como peso não seria o ideal, pois o erro cometido estaria sendo distribuído entre as seções onde se obteve desníveis mais precisos. Então se optou por utilizar como peso o inverso do erro de fechamento de cada seção. Deste modo, seções com melhor fechamento possuem os maiores pesos. Também se optou por tratar o nivelamento e contranivelamento como observações separadas. Desta forma, o circuito de nivelamento é configurado como uma rede (Figura 11).

Com:

$\mathrm{n}^{\mathrm{o}}$ de observações $=8$

$\mathrm{n}^{\mathrm{o}}$ de incógnitas $=3$

$\mathrm{n}^{\mathrm{o}}$ de equações de condição $=5$ 
Figura 11 - Ajustamento pelo método dos correlatos.

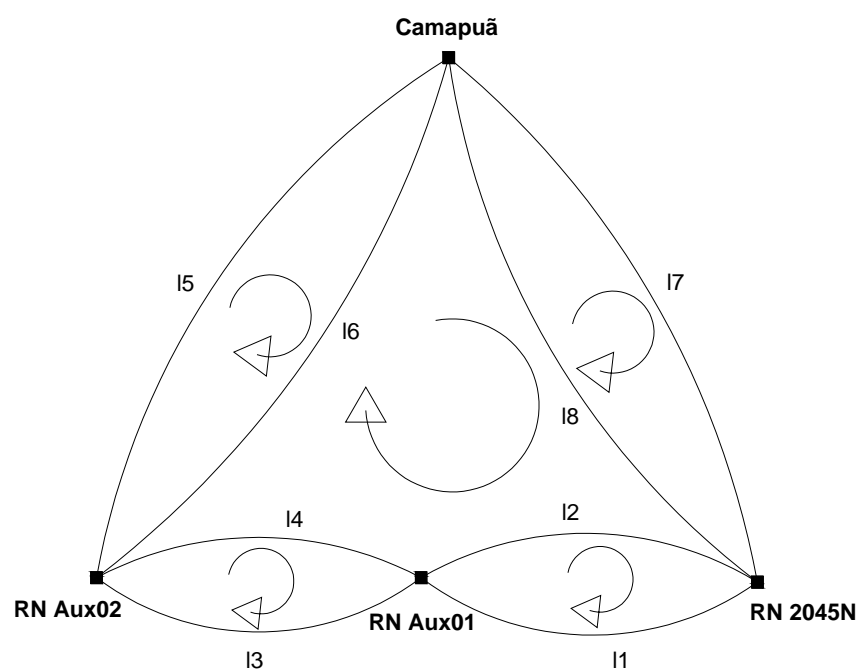

No Quadro 8 pode-se observar os desníveis medidos ( $\mathrm{Lb}$ ), e os resultados do ajustamento, com o vetor dos resíduos (V) e o vetor dos desníveis ajustados (La).

Quadro 8 - Ajustamento - observações, resíduos e valores ajustados.

\begin{tabular}{|c|c|c|}
\hline $\mathrm{Lb}(\mathrm{m})$ & $\mathrm{V}(\mathrm{m})$ & $\mathrm{La}(\mathrm{m})$ \\
\hline 8,2566 & 0,0013 & 8,2579 \\
$-8,2556$ & $-0,0023$ & $-8,2579$ \\
$-23,3623$ & 0,0080 & $-23,3543$ \\
23,3592 & $-0,0050$ & 23,3543 \\
910,6316 & 0,0322 & 910,6638 \\
$-910,6081$ & $-0,0557$ & $-910,6638$ \\
$-895,5808$ & 0,0134 & $-895,5674$ \\
895,5906 & $-0,0232$ & 895,5674 \\
\hline
\end{tabular}

Pode-se notar que os resíduos são menores para as seções de nivelamento ao longo da rodovia, onde se obteve melhor fechamento de seção, como era de se esperar, considerando a forma como foi configurada a matriz dos pesos. 
A seguir é apresentada a matriz variância-covariância dos desníveis ajustados:

$$
\Sigma \mathrm{La}=\left[\begin{array}{rrrrrrrr}
0,0005 & -0,0005 & 0,0000 & 0,0000 & -0,0003 & 0,0003 & -0,0001 & 0,0001 \\
-0,0005 & 0,0005 & 0,0000 & 0,0000 & 0,0003 & -0,0003 & 0,0001 & -0,0001 \\
0,0000 & 0,0000 & 0,0016 & -0,0016 & -0,0011 & 0,0011 & -0,0004 & 0,0004 \\
0,0000 & 0,0000 & -0,0016 & 0,0016 & 0,0011 & -0,0011 & 0,0004 & -0,0004 \\
-0,0003 & 0,0003 & -0,0011 & 0,0011 & 0,0044 & -0,0044 & -0,0031 & 0,0031 \\
0,0003 & -0,0003 & 0,0011 & -0,0011 & -0,0044 & 0,0044 & 0,0031 & -0,0031 \\
-0,0001 & 0,0001 & -0,0004 & 0,0004 & -0,0031 & 0,0031 & 0,0036 & -0,0036 \\
0,0001 & -0,0001 & 0,0004 & -0,0004 & 0,0031 & -0,0031 & -0,0036 & 0,0036
\end{array}\right]
$$

Analisando a matriz variância-covariância, observa-se melhor precisão para as seções de nivelamento ao longo da BR116, onde se obteve melhor fechamento de seção. No Quadro 9 pode-se observar a altitude ajustada do Pico do Camapuã.

Quadro 9- Altitude ajustada do pico do Camapuã.
\begin{tabular}{|c|c|}
\hline \multicolumn{2}{|c|}{ Altitude } \\
\hline Pico do Camapuã & $\mathbf{1 . 7 1 1 , 9 5 m}$ \\
\hline
\end{tabular}

\section{CONCLUSÕES E RECOMENDAÇÕES}

$\mathrm{Na}$ determinação dos desníveis entre as RN's ao longo da rodovia BR116, utilizando nivelamento trigonométrico pelo método Leap-Frog, mesmo com visadas de comprimentos superiores aos recomendados pela literatura, e com a estação total instalada fora do centro do lance, conseguiu-se fechamento de alta precisão, segundo a Resolução PR $\mathrm{n}^{\circ} 22$ (IBGE, 1983). Atribui-se isso ao equipamento utilizado (estação total Leica TC2003), com precisão linear de $1 \mathrm{~mm}+1 \mathrm{ppm}$ e angular de 0,5 ", e a acuidade visual do operador, proporcionando pontarias com repetibilidade da ordem de 2", mesmo em condições de alto tráfego da rodovia. Em certos momentos foi necessário interromper as medições devido à vibração causada pelo tráfego intenso de caminhões pesados.

Ainda com relação ao desnível entre as RN's às margens da rodovia, pôde-se

perceber que a influência da refração quando se utiliza este método de nivelamento é significativa. Para a seção de nivelamento realizada entre a RN2045N e RN Aux01, ocasião em que o tempo estava nublado, obteve-se fechamento melhor do que para a seção entre as RN's Aux01 e Aux02, realizado em um dia com forte insolação.

No nivelamento trigonométrico entre as RN's às margens da rodovia e o cume do Camapuã, não foi possível realizar o número de séries PD-PI necessárias para se atingir a precisão estimada de $3 \mathrm{~mm} \sqrt{\mathrm{k}}$ considerando a propagação de erros em 
função da precisão nominal do equipamento. Isto ocorreu devido às dificuldades encontradas em se realizar os levantamentos de campo nas condições propostas neste trabalho, tendo-se realizado o maior número possível de séries dentro destas condições.

Ainda com relação ao transporte de altitudes para o cume do Camapuã, efetuaram-se duas seções de nivelamento partido de RN's distintas, possibilitando o fechamento de um circuito. Numa primeira seção, partindo da RN2045N, obteve-se fechamento de $4 \mathrm{~mm} \sqrt{\mathrm{k}}$, considerado como de precisão segundo a Resolução $\mathrm{PR} \mathrm{n}^{\circ}$ 22 do IBGE. Este resultado foi considerado muito satisfatório levando em consideração o número de séries PD-PI realizadas, que foi bem abaixo do número calculado em função da precisão nominal do equipamento. Para a seção realizada a partir da RN Aux02, o fechamento foi de $12 \mathrm{~mm} \sqrt{\mathrm{k}}$ (para fins topográficos segundo a Resolução PR $\mathrm{n}^{\circ}$ 22), um resultado satisfatório função do número de série realizadas.

Outro problema encontrado no transporte de altitudes entre as RN's as margens da rodovia e o cume da montanha foi o feito da refração atmosférica e da reverberação. No intervalo entre $10 \mathrm{~h}$ e $15 \mathrm{~h}$ é praticamente inviável a aplicação desta metodologia.

Tendo em vista os resultados alcançados, tanto no nivelamento ao longo da rodovia, como no nivelamento para o cume do Camapuã, pode-se considerar a metodologia utilizada como eficaz em função das condições adversas encontradas durante a execução deste trabalho.

Com respeito à refração, recomenda-se a realização do nivelamento trigonométrico no período noturno, utilizando o alvo com sistema de iluminação desenvolvido durante a pesquisa, e se possível, realizando um número maior de séries PD-PI, na tentativa de se atingir fechamento de alta precisão.

\section{AGRADECIMENTOS}

Os autores agradecem a CAPES - Coordenação de aperfeiçoamento de Pessoal de Nível Superior pelo apoio financeiro em forma de bolsa de estudos concedida ao autor Daniel Perozzo dos Santos.

Os autores também agradecem aos amigos Niarkios, Kanke, Rodrigo, Emídio, César, Vitor, Wander, Rógers, Gracianne, Marcos, Sílvio e Wilson, que muito contribuíram na execução dos levantamentos de campo.

\section{REFERÊNCIAS BIBLIOGRÁFICAS}

CEYLAN, A. ; BAYCAL, O. Precise Height Determination Using Simultaneous Reciprocal Trigonometric Levelling. Survey Review, 40, 308 pp195-205, April, 2008.

CEYLAN, A. ; INAL, C. ; SANLIOGLU, I. Modern Height Determination Thechniques and Comparison of Accuracies. FIG Working Week, Cairo, Egipt, 2005 . 
FAGGION, P. L.; VEIGA, L. A. K.; FREITAS, S. R. C.; SANTOS, D. P. Desníveis de Primeira Ordem com Estação Total. Série em Ciências Geodésicas, volume 3, Curso de Pós-Graduação em Ciências Geodésicas, Departamento de Geomática, Setor de Ciências da Terra, UFPR. Curitiba, 2003.

FAGGION, P. L. Considerações sobre a instrumentação topográfica, Curso de Pós-Graduação em Ciências Geodésicas, Departamento de Geomática, Setor de Ciências da Terra, UFPR. Curitiba, 2006.

GEMAEL, CAMIL. Introdução ao Ajustamento de Observações: Aplicações Geodésicas. Editora UFPR, Curitiba, 1994.

GOMES, J. P. Determinação de Desníveis de Precisão Utilizando Estação Total Dissertação de Mestrado, Curso de Pós-Graduação em Ciências Geodésicas, Setor de Ciências da Terra, UFPR, Curitiba, 2006.

IBGE. Resolução PR n ${ }^{\circ} 22$ de 21/07/1983.

KAHMEN, H. ; FAIG, W. Surveying. Berlin/New York, Walter de Gruyter, 1988.

MOREIRA, A. S. B. Nivelamento Trigonométrico e Nivelamento Geométrico Classe IIN da NBR 13.133: Limites e condições de compatibilidade. Dissertação de Mestrado, Escola de Engenharia de São Carlos, Universidade de São Paulo, São Carlos, 2003.

RÜEGER, J. M. Eletronic Distance Measurement, 4 ${ }^{\mathrm{a}}$ edição. Springer-Vervag. Berlin Heidelberg, 1996.

Recebido em junho de 2010. Aceito em junho de 2011. 